\title{
Early identification of Mycobacterium tuberculosis complex in BACTEC cultures by ligase chain reaction
}

\author{
S. X. WANG and L. TAY \\ Central Tuberculosis Laboratory, Department of Pathology, Singapore General Hospital, Republic of Singapore
}

\begin{abstract}
A total of 1431 acid-fast bacilli (AFB) in Bactec $^{\circledR}$ culture vials from 1427 patients was differentiated by the Bactec NAP ${ }^{\circledR}$ method and tested by the LCx Mycobacterium tuberculosis ligase chain reaction system. In all, 1321 of 1325 M. tuberculosis complex (MTBC) isolates were correctly detected by the LCx assay. All the 106 non-tuberculous mycobacteria (NTM) isolates were negative by the LCx assay. No false MTBC-positive result was obtained from NTM isolates. However, the $\mathrm{LCx}$ assay failed to detect four MTBC isolates from one patient. Sensitivity, specificity, positive predictive value (PPV) and negative predictive value (NPV) were $99.7,100 \%, 100 \%$ and $96.4 \%$, respectively. These data suggest that the LCx system can be used to identify MTBC in AFB-positive Bactec broth cultures when the growth index is $\geqslant 100$. The method gives a $100 \%$ PPV and allows a faster turnaround time for MTBC than the NAP test.
\end{abstract}

\section{Introduction}

Rapid diagnosis of tuberculosis is an important component of measures to control the disease [1], which is an objective made more urgent by the appearance of multidrug-resistant strains [2]. Nucleic acid amplification techniques, which allow detection of Mycobacterium tuberculosis complex (MTBC) organisms directly from samples in a matter of hours, apparently represent the current best answer to this issue. However, the relatively low sensitivity of these techniques for smear-negative specimens restricts their use to selected sample types, primarily respiratory samples. Culture confirmation remains essential, as nucleic acid amplification techniques are unable to differentiate live from dead mycobacteria. The Bactec ${ }^{\circledR}$ radiometric method (Becton Dickinson, Towson, MD, USA) is one of the fastest and most sensitive cultural tools for the diagnosis of mycobacterial infections $[3,4]$. The sensitivity of the radiometric assay allows a culture to reach a positive threshold in a very short time, often within 1-2 weeks. Confirmation of the presence of acid-fast bacilli (AFB) in a positive Bactec vial can be achieved by microscopy. However, a microscopically confirmed positive culture may be

Received 20 Sept. 2001; revised version accepted 4 April 2002.

Corresponding author: Dr Tay Leng (e-mail: gpttay@sgh. com.sg). either MTBC or a non-tuberculous mycobacterium (NTM), which poses a dilemma both for therapy and for the measures to be adopted to prevent the spread of infection. Furthermore, differentiation of MTBC from NTM by the NAP ( $\rho$-nitro- $\alpha$-acetylamino- $\beta$-hydroxypropiophenone) test requires $4-8$ days to give a result. A procedure enabling early identification of MTBC in a positive Bactec culture vial would be valuable.

This study investigated the reliability of a commercial ligase chain reaction system (LCx M. tuberculosis; Abbott Diagnostic Division, Abbott Park, IL, USA) $[5,6]$ for the detection of MTBC in AFB-positive Bactec culture vials.

\section{Materials and methods}

Bactec $12 \mathrm{~B}^{\circledR}$ cultures showing a minimum growth index (GI) of $>50$ were selected initially for inclusion in the study. An AFB smear was performed and, if positive, a NAP test was performed by standard methods [7]. For those vials in which no AFB were seen and for the $\mathrm{LCx}$ procedure, incubation was continued for at least 1 more day to produce a GI of $\geqslant 100$ with a positive AFB smear. In practice, GIs of cultures used in this study ranged from 100 to 999. Each broth culture that was differentiated as NTM by NAP was subcultured on solid medium and the mycobacterial species was identified by conventional 
biochemical tests and commercial DNA probes (AccuProbe, San Diego, CA, USA). A total of 1431 Bactec 12B broth cultures from 1427 patients with GI of $\geqslant 100$ was subjected to the LCx assay following the procedure recommended by the manufacturer for clinical samples. Briefly, $500 \mu \mathrm{l}$ of each Bactec broth were transferred to a screw-capped microcentrifuge tube containing respiratory specimen buffer. The tube was vortex mixed and centrifuged (1500 $\mathrm{g}$ for $10 \mathrm{~min}$ ) and the resulting pellet was washed and resuspended in buffer. After inactivation at $95^{\circ} \mathrm{C}$ for $20 \mathrm{~min}$, the suspension was cooled to room temperature and lysed for $10 \mathrm{~min}$ in an Abbott Lysor sonicator. After further centrifugation (9000 $\mathrm{g}$ for $2 \mathrm{~min}$ ), $100 \mu \mathrm{l}$ of supernate were transferred to a tube containing the amplification mixture. Amplification was performed in a thermal cycler (LCx Thermal Cycler) for 37 cycles as follows: $94^{\circ} \mathrm{C}$ for $1 \mathrm{~s}, 64^{\circ} \mathrm{C}$ for $1 \mathrm{~s}$, and $69^{\circ} \mathrm{C}$ for $40 \mathrm{~s}$ with maintenance at $25^{\circ} \mathrm{C}$ at the end of the last cycle. For each series of tests, the negative control and calibrator provided were prepared in duplicate and subjected to the same amplification procedure as the samples. Amplified tubes were pulse-centrifuged (10-15 s) and transferred unopened to the carousel of the LCX analyser, which directly detects amplification products by a microparticle enzyme immunoassay, reporting the results as fluorescence rates that are compared to the calibrator rate. Results $>30 \%$ of the average of the calibrator rate were considered positive.

\section{Results and discussion}

Of the 1321 cultures that were positive for MTBC by the LCx assay, 1320 were identified as MTBC by NAP. The remaining sample proved to be a mixture of MTBC and M. kansasii. Of 110 mycobacterial cultures negative for MTBC by the LCx assay, 106 were identified as NTM by NAP (22 M. avium complex, 21 M. kansasii, 10 M. gordonae, 9 M. chelonae species group, $6 \mathrm{M}$. marinum, $5 \mathrm{M}$. fortuitum species group, 2 $M$. haemophilium and 31 other NTMs). The four MTBC isolates found to be LCX-negative were subcultured on solid medium and confirmed as MTBC by conventional biochemical tests plus commercial MTBC DNA probe. These four MTBC isolates were from samples from a single patient. No false-positive results were obtained. Sensitivity, specificity, positive predictive value (PPV) and negative predictive value (NPV) were, therefore, $99.7 \%, 100 \%, 100 \%$ and $96.4 \%$, respectively.

Several previous studies have evaluated the possibility of rapid detection of MTBC in early-positive Bactec vials. In the majority of these, the commercial AccuProbe was used [8-10]; the reported sensitivities ranged from $33 \%$ to $83 \%$. In only one study were nine of nine positive cultures detected [11]. A commercial amplification assay (Amplicor MTB; Roche Molecular Systems, Somerville, NJ, USA) has also been investigated for the same purpose; the sensitivities obtained ranged from $66 \%$ to $100 \%[12-14]$. In one study, an in-house PCR achieved better sensitivity and specificity results than a commercially available PCR product [14]. However, such procedures are only suitable for specialised laboratories, as they require dedicated personnel and facilities. The manufacturer does not recommend the use of the LCx system for the detection of MTBC from positive Bactec culture vials. However, the results of this study have shown that there is a good correlation between NAP and LCx results. The four isolates from a single patient that gave falsenegative LCx results could be due to gene mutation or deletion of the LCx target nucleic acid sequence $[15,16]$. These data suggest that the LCx system can be used to identify MTBC in AFB-positive Bactec broth cultures when the GI is $\geqslant 100$. The method gives a $100 \%$ PPV and allows a faster turnaround time for MTBC than the NAP test.

We thank Ms Hla Hla Htay and Ha Kee Mong for their technical assistance.

\section{References}

1. Tenover FC, Crawford JT, Huebner RE, Geiter LJ, Horsburgh $\mathrm{CR}$, Good RC. The resurgence of tuberculosis: Is your laboratory ready? J Clin Microbiol 1993; 31: 767-770.

2. Perronne C. Multiple-drug-resistant tuberculosis. Current aspects in industrialized countries, and future strategies. Res Microbiol 1993; 144: 129-133.

3. Pfyffer GE, Welscher H-M, Kissling P et al. Comparison of the Mycobacteria Growth Indicator Tube (MGIT) with radiometric and solid culture for recovery of acid-fast bacilli. J Clin Microbiol 1997; 35: 364-368.

4. Roggenkamp A, Hornef MW, Masch A, Aigner B, Autenrieth IB, Heesemann J. Comparison of MB/BacT and BACTEC 460 TB systems for recovery of mycobacteria in a routine diagnostic laboratory. J Clin Microbiol 1999; 37: 3711-3712.

5. Lumb R, Davies K, Dawson D et al. Multicenter evaluation of the Abbott LCx Mycobacterium tuberculosis ligase chain reaction assay. J Clin Microbiol 1999; 37: 3102-3110.

6. Wang SX, Tay L. Evaluation of three nucleic acid amplification methods for direct detection of Mycobacterium tuberculosis complex in respiratory specimens. J Clin Microbiol 1999; 37: $1932-1934$

7. Siddiqi SH. BACTEC460 ${ }^{\circledR}$ TB SYSTEM. 1995; Products and Procedure Manual. Revision D.

8. Body BA, Warren NG, Spicer A, Henderson D, Chery M. Use of Gen-Probe and Bactec for rapid isolation and identification of mycobacteria. Correlation of probe results with growth index. Am J Clin Pathol 1990; 93: 415-420.

9. Reisner BS, Gatson AM, Woods GL. Use of Gen-Probe AccuProbes to identify Mycobacterium avium complex, Mycobacterium tuberculosis complex, Mycobacterium kansasii, and Mycobacterium gordonae directly from BACTEC TB broth cultures. J Clin Microbiol 1994; 32: 2995-2998.

10. Telenti M, De Quiros JFB, Alvarez M, Rionda MJS, Mendoza MC. The diagnostic usefulness of a DNA probe for Mycobacterium tuberculosis complex (Gen-Probe) in BACTEC cultures versus other diagnostic methods. Infection 1994; 22: 18-23.

11. Chapin-Robertson K, Dahlberg S, Waycott S, Corrales J, Kontnick C, Edberg SC. Detection and identification of Mycobacterium directly from BACTEC bottles by using a DNA-rRNA probe. Diagn Microbiol Infect Dis 1993; 17: 203-207.

12. Smith MB, Bergmann JS, Woods GL. Detection of Mycobacterium tuberculosis in BACTEC 12B broth cultures by the Roche Amplicor PCR assay. J Clin Microbiol 1997; 35: 900-902.

13. Ninet B, Rohner P, Metral C, Auckenthaler R. Assessment of 
use of the COBAS AMPLICOR system with BACTEC 12B cultures for rapid detection of frequently identified mycobacteria. J Clin Microbiol 1999; 37: 782-784.

14. Eing BR, Becker A, Sohns A, Ringelmann R. Comparison of Roche Cobas Amplicor Mycobacterium tuberculosis assay with in-house PCR and culture for detection of $M$. tuberculosis. J Clin Microbiol 1998; 36: 2023-2029.
15. Andersen AB, Hansen EB. Structure and mapping of antigenic domains of protein antigen b, a 38,000-molecular-weightprotein of Mycobacterium tuberculosis. Infect Immun 1989; 57: 2481-2488.

16. Sjöbring U, Mecklenburg M, Andersen ÅB, Miörner $\mathrm{H}$ Polymerase chain reaction for detection of Mycobacterium tuberculosis. J Clin Microbiol 1990; 28: 2200-2204. 